\title{
Experiencia de trombolisis sistematizada en infarto cerebral agudo en un hospital público de Chile
}

'Servicio de Neurología Hospital Barros Luco Trudeau, Santiago, Chile. Departamento Neurología Campus Sur, Facultad de Medicina Universidad de

Chile, Santiago, Chile. ${ }^{2}$ Facultad de Medicina Clínica AlemanaUniversidad del Desarrollo. Santiago, Chile.

aMaster en Bioestadística. Recibido el 28 de septiembre de 2010, aceptado el 21 de junio de 2011.

Correspondencia a:

Dra. Tatiana Figueroa Reyes Servicio de Neurología Hospital Barros Luco Trudeau Santiago Chile, Departamento Neurología, Facultad de Medicina, Universidad de Chile. Av. José Miguel Carrera 4700 San Miguel, Santiago, Chile. Fono: 3948941

E.-mail: tfigueroa@med. uchile.cl
TATIANA FIGUEROA-REYES ${ }^{1}$, DAVID SÁEZ M. ${ }^{1}$, ELOY MANSILLA L. ${ }^{1}$, RODRIGO SÁNCHEZ V. ${ }^{1}$, JORGE NOGALES-GAETE ${ }^{1}$, IRIS DELGADO B. 2,a

\section{Thrombolysis for acute ischemic stroke with recombinant tissue plasminogen activator in a Chilean public hospital}

Background: The only accepted treatment for acute ischemic stroke is thrombolysis with recombinant tissue plasminogen activator ( $t$-PA). It was implemented in Chile in 1996, although its use was mainly restricted in Chile to private clinics. Recently, at year 2009, we have implemented this treatment in a public hospital. Aim: To describe the results of treatment of acute ischemic stroke with $t$-PA in a public hospital in Chile. Material and Methods: Prospective analysis of all eligible patients with acute ischemic stroke that were admitted within 4 hours of its onset and had no contraindications for thrombolysis. Results: In an eight months period, a total of 19 intravenous thrombolyses were performed in 12 males and seven females aged 28 to 79 years old. The mean lapse between onset of symptoms and onset of thrombolysis was $190 \pm 57$ min. Results were favorable, according to Rankin and National Institute of Health Stroke scales. Ninety days after treatment, 63\% of patients had minimal or absent disability, 26\% had moderate disability and only one (5\%) had severe disability. One patient had a clinically not significant intracranial hemorrhage and one patient died six days after thrombolysis. Conclusions: These results indicate that thrombolysis can be successfully implemented in Chilean public hospitals. The limitations for its use in this setting are mostly administrative.

(Rev Med Chile 2011; 139: 1118-1127).

Key words: Plasminogen activators; Stroke; Thrombolytic therapy.
E n Chile la incidencia de un primer infarto cerebral se estima en 100 por 100.000 habitantes por año ${ }^{1}$, con 10.435 egresos hospitalarios en 2006 por esta causa ${ }^{2}$.

La Enfermedad Cerebrovascular (ECV) es la patología que presenta mayor carga de enfermedad por causa específica en mayores de 74 años, medidos en AVISA (años de vida saludable perdidos ajustados por discapacidad) ${ }^{3}$. El $93 \%$ de los infartos cerebrales se produce en mayores de 45 años, con letalidad de $19 \%$ al mes y $28 \%$ a seis meses $^{1}$. Sin embargo, el mayor impacto en AVISA está dado por el factor discapacidad, $18 \%$ a seis meses $^{3}$ en grado moderado a severo. En este sentido la trombolisis logra disminuir la discapacidad a largo plazo ${ }^{4-9}$.

Hasta ahora, ningún hospital público chileno realizaba trombolisis sistemáticamente pese a ser una recomendación en la guía de garantías explícitas en salud $(\mathrm{GES})^{10}$, sistema de atención que norma acceso, oportunidad, calidad y protección financiera.

El Complejo Asistencial Barros Luco (CABL), es un hospital público, de alta complejidad, ubicado en la zona sur de Santiago, cuya población beneficiaria es 1.043.013 habitantes. El Servicio de 
Trombolisis en infarto cerebral agudo - T. Figueroa-Reyes et al

Neurología es a su vez, Departamento de la Universidad de Chile, centro formador de neurólogos.

Nuestro Servicio realizó el año 1998 una propuesta para la atención de pacientes con ECV en etapa aguda ${ }^{11}$, definiendo como prioritaria su atención dado el importante empleo de recursos y la morbi-mortalidad asociada ${ }^{12}$. El 2009 hubo 599 egresos por infarto cerebral, con $8,7 \%$ de letalidad.

El objetivo del estudio es describir las condiciones de recursos humanos, capacitación y gestión que hacen posible la implementación de trombolisis endovenosa en un hospital público, los tiempos de las diferentes etapas del proceso y las características demográficas, clínicas y de evolución de los pacientes trombolizados.

\section{Pacientes y Método}

El 2009 se estudiaron las condiciones administrativas, organizacionales y de gestión necesarias para implementar trombolisis en nuestro hospital. Esto permitió diseñar un flujograma de atención consensuado por los distintos estamentos y Servicios involucrados en el procedimiento.

La estrategia y gestión abarcó la capacitación del recurso humano prehospitalario y hospitalario del Servicio de Urgencia (SU), para el reconocimiento del ictus mediante la Escala de Cincinnati ${ }^{13}$. En Atención Hospitalaria se capacitó en trombolisis a los diferentes estamentos de los Servicios de Urgencia, Neurología y Laboratorio. Neurólogos y residentes fueron entrenados en Escala del National Institute of Health Stroke Scale (NIHSS $)^{14,15}$ y en reconocimiento de signos precoces de infarto en tomografía cerebral (TC) según criterios de Alberta Stroke Program Early CT Score (ASPECTS) ${ }^{16-18}$. Se diseñó y distribuyó en los Servicios pertinentes material que incluyó tríptico con Escala NIHSS, afiche con flujograma y ficha de enfermería específica. La gestión de Infraestructura, consideró el recurso cama para realizar trombolisis en la Unidad Cerebro Vascular (UCV) del Servicio de Neurología ${ }^{11}$.

Los pacientes con síntomas sugerentes de ictus agudo en ventana, son identificados por personal de enfermería en admisión de SU, simultáneamente comunica al equipo de neurología de turno. Este último solicita TC y exámenes de laboratorio según flujograma (Figura 1). Los criterios de inclusión y exclusión ${ }^{9}$ se muestran en Tablas 1 y 2. En todos los pacientes se registraron tiempos de: 1) inicio de síntomas; 2) llegada a admisión de SU; 3 ) categorización de enfermería (triage); 4) primera evaluación por neurólogo; 5) latencia de laboratorio; 6) latencia de tomografía y 7) inicio de trombolisis.

Se realizó TC sin contraste en todos los pacientes. Se aplicaron criterios ASPECTS para detección de signos precoces de infarto cerebral ${ }^{16-18}$. La tomografía fue evaluada por el equipo de neurología de turno. Previo a la trombolisis todos los pacientes con infarto de territorio posterior y casos seleccionados de circulación anterior, fueron estudiados con resonancia magnética (RM) protocolo Stroke, que incluye secuencias en T2, T2 GRE, Difusión, mapa ADC y Flair, el criterio de discriminación estuvo dado por la gravedad inicial de los pacientes e incertidumbre diagnóstica. Se obtuvo consentimiento informado en todos los casos. Se decidió realizar trombolisis en el Servicio de Neurología, para optimizar el tiempo puerta- aguja, el que se prolongaba al realizar el procedimiento en SU, esto debido a la carga asistencial y la limitada disposición de camas de manera rápida en un Servicio de alto flujo.

Se administró Actilyse (activador del plasminógeno tisular recombinante humano) (rt-PA) a dosis $0,9 \mathrm{mg} / \mathrm{kg}$, dosis máxima de $90 \mathrm{mg}$. Los pacientes fueron evaluados con Escala de Rankin modificada $^{19}$, que mide discapacidad, con puntajes entre 0-6 y Escala de NIHSS ${ }^{14,15}$, al ingreso, 24 horas, egreso, 30 y 90 días. La evaluación al alta fue realizada por un neurólogo distinto al que realizó el procedimiento, todos los controles fueron presenciales. Todos los pacientes se trataron según protocolo, excepto dos infartos de fosa posterior con NIHSS de ingreso de 29 y 33 puntos.

Se realizó TC de control a las 24 horas, siete días postrombolisis o antes, en caso de deterioro clínico. El estudio etiológico realizado posterior al procedimiento incluyó: RM de cerebro, Doppler cervical, angioTC de vasos cervicales e intracraneanos, ecocardiograma transtorácico y/o transesofágico, Holter de ritmo; en casos seleccionados se realizó angioRM de vasos cervicales, angiografía convencional, perfil inmunológico y estudio de trombofilia.

Los subtipos de infarto fueron clasificados según criterios TOAST ${ }^{20}$ y de Oxfordshire Community Stroke Project ${ }^{21}$.

En el período de estudio comprendido entre septiembre de 2009 y abril de 2010, ingresaron 


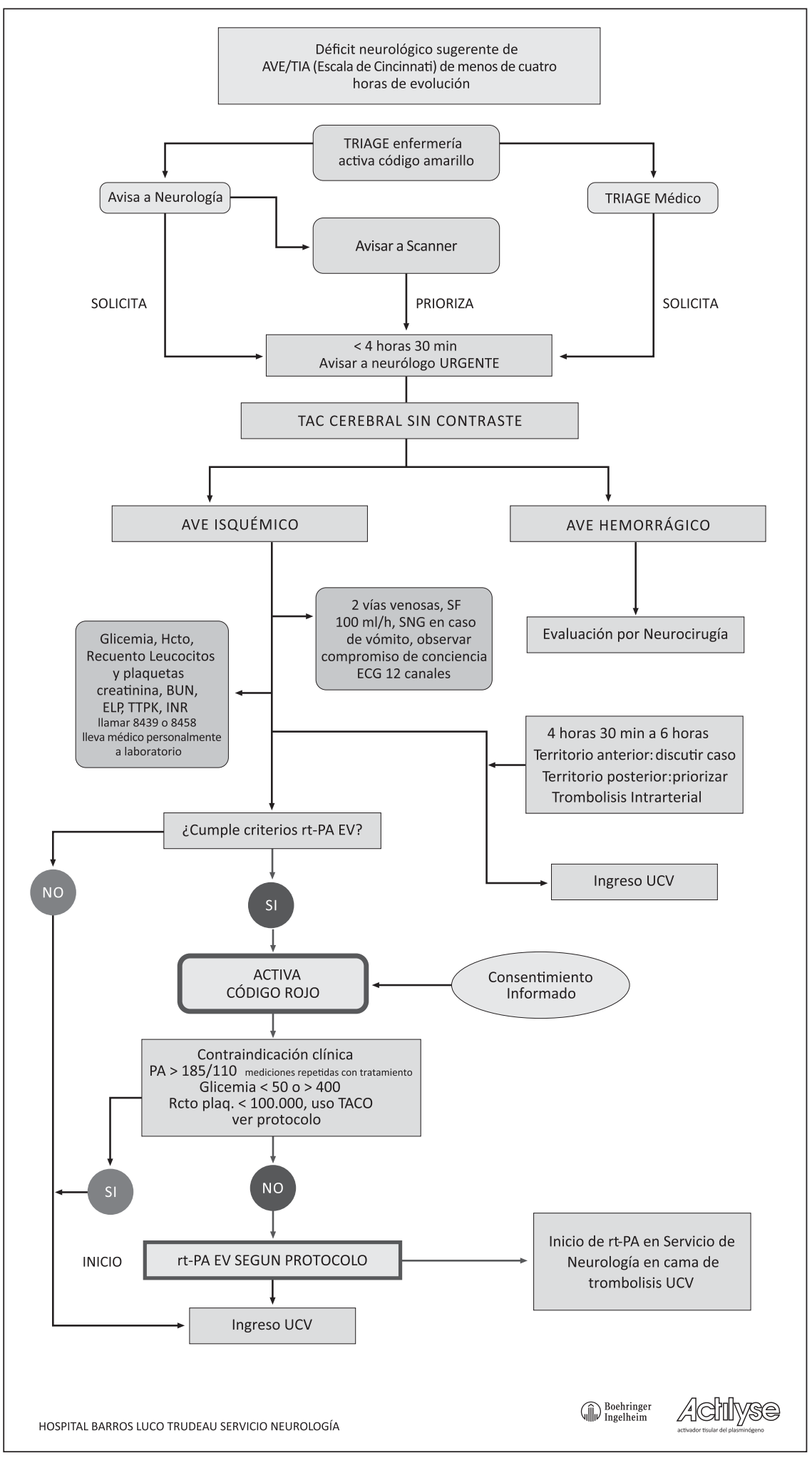

Figura 1. Flujograma del proceso de trombolisis de pacientes con AVC ingresados. 
Trombolisis en infarto cerebral agudo - T. Figueroa-Reyes et al

\section{Tabla 1. Criterios de inclusión}

Infarto cerebral de menos de 270 minutos de evolución, con hora de inicio claramente definida

Déficit neurológico de más de 4 puntos en la escala de NIHSS y presentes por más de 30 minutos, sin mejoría previo al inicio de la infusión

Edad entre 18 y 79 años

Tomografía computarizada de cerebro sin evidencias de hemorragia cerebral

No presentar criterios de exclusión

\section{Tabla 2. Criterios de exclusión}

Hora de inicio desconocida, o más de 270 minutos al inicio de la infusión

Déficit neurológico severo evaluado clínicamente (NIHSS $>23$ ), o por técnica de neuroimagen (ASPECT Score $<7$ ), excepto en infartos de circulación posterior

Infarto o traumatismo craneal en los últimos 3 meses

Cirugía mayor los últimos 14 días

Antecedente de hemorragia intracraneal

Presión arterial sistólica $>180$ o diastólica $>110 \mathrm{mmHg}$ en mediciones repetidas

Necesidad de tratamiento antihipertnesivo agresivo para llegar a esos niveles

Rápido mejoramiento de los síntomas

Síntomas sugerentes de hemorragia subaracnoídea, incluso con Tomografía normal

Antecedente de hemorragia gastrointestinal o urinaria en los últimos 21 días

Punción arterial en sitio no susceptible de compresión

Insuficiencia hepática, renal, cardiaca severa

Antecedente de ECV previo en paciente con Diabetes Mellitus. Retinopatía diabética proliferativa

Crisis convulsivas al inicio de los síntomas

Uso de anticoagulantes

Tiempo de protrombina $>15$ segundos

Uso de heparina 48 horas previas al inicio de los síntomas, con TTPK elevado

Glicemia $<50$ o $>400$ mg/dl al SU 390 infartos cerebrales, de los cuales 25 $(6,4 \%)$ llegaron en período de ventana, se realizó trombolisis a 19 pacientes. Seis fueron excluidos por las siguientes causas: dos por trauma craneal, uno en anticoagulación, uno por pruebas de laboratorio alteradas, uno por ASPECTS bajo 6 y uno por edad.

Los datos de todos los pacientes se consignaron en base ACCESS. Se realizó un análisis descriptivo utilizando números absolutos y porcentajes para las variables categóricas; para las continuas, se calcularon estadísticas de dispersión y tendencia central. Las asociaciones estadísticas se verificaron a través de $\chi^{2}$ para las variables categóricas y test de Friedman para la comparación entre las medianas de escala de NIHSS. Se consideró significación estadística para un valor de $\mathrm{p}<0,05$. Todos los análisis estadísticos se realizaron en SPSS versión 18.0.

\section{Resultados}

De los 19 pacientes tratados la edad promedio fue 56,8 años ( $\mathrm{DE} \pm 13,5)$, rango 28 a 79 años. La distribución por sexo fue 12 hombres $(63,2 \%)$ y siete mujeres. El promedio de edad de los hombres fue de 61 años, en mujeres 50.

El tiempo promedio transcurrido entre el inicio de síntomas e inicio de trombolisis fue 197,8 minutos $(\mathrm{DE} \pm 56,6)$, mínimo de 81 y máximo 270 minutos.

El promedio del tiempo transcurrido entre inicio de síntomas hasta la llegada a urgencias fue de 87 minutos, ( $\mathrm{DE} \pm 46,8$ minutos), con un rango de 30 a 180. Dos pacientes llegaron con una demora mayor de 2 horas (170 y 176 minutos), ambos derivados de otro Hospital, ingresando con TC y exámenes de laboratorio realizados. La Figura 2, muestra para cada uno de los pacientes los tiempos trascurridos entre: 1) inicio de síntomas hasta llegada a Urgencia; 2) Urgencia y Categorización; 3) Categorización e inicio de Trombolisis. Se observa, en todos los casos, que el tiempo total entre inicio de síntomas e inicio de trombolisis fue menor a 270 minutos. El tiempo promedio entre llegada a Urgencia y categorización fue 9 minutos ( $\mathrm{DE} \pm 6,6)$, entre categorización e inicio de trombolisis 102 minutos ( $\mathrm{DE} \pm 41,8)$ con un tiempo mínimo de 45 y un máximo de 175 minutos, lo que arroja un tiempo puerta-aguja promedio de 111 minutos. La latencia de realización de TC prome- 


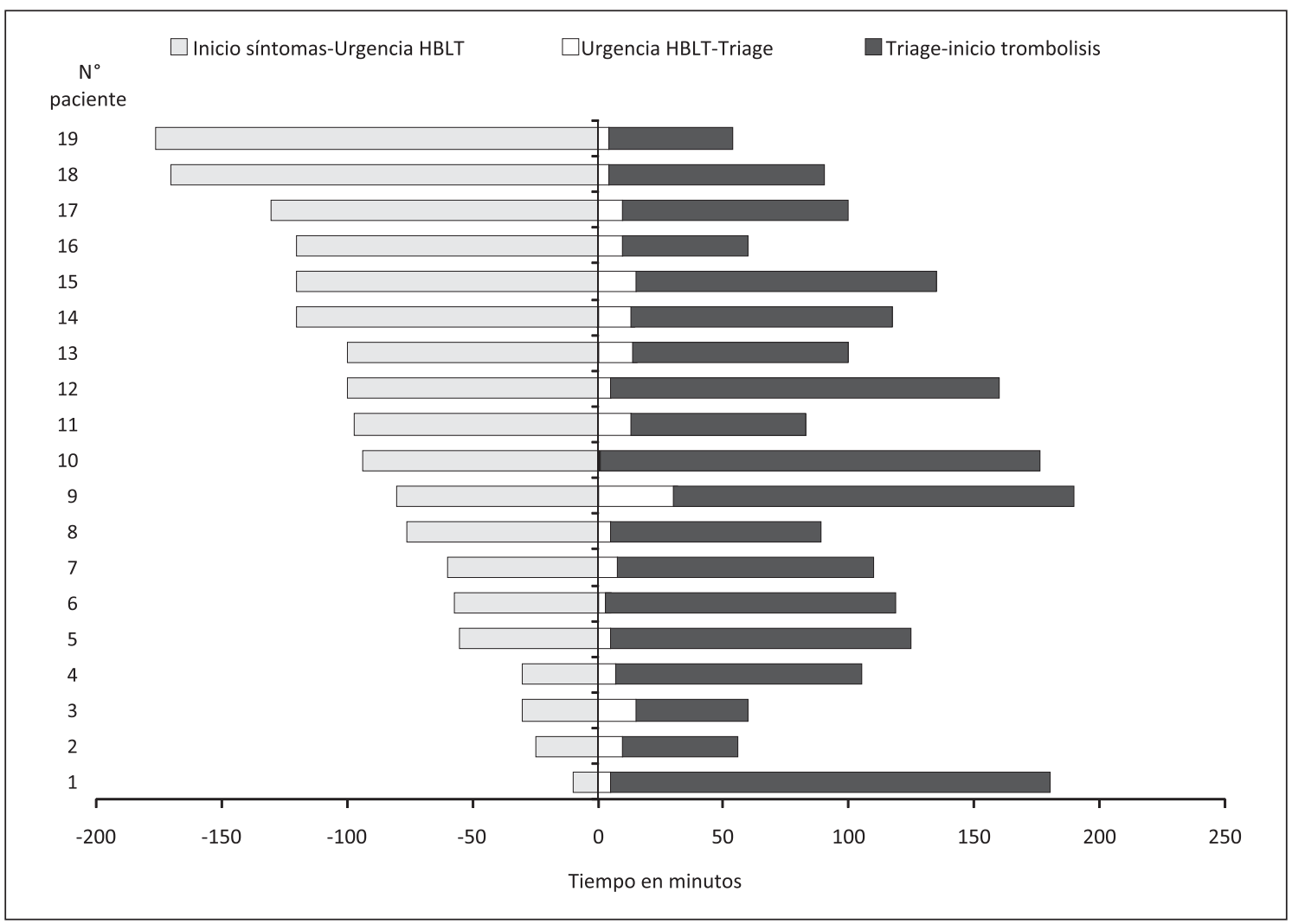

Figura 2. Tiempos entre los distintos momentos desde el inicio de síntomas hasta el inicio de trombolisis endovenosa.

dio fue 15 minutos y el de exámenes de laboratorio de 30 minutos. El tiempo de categorización-inicio de trombolisis se reduce, puesto que se realizan actividades simultáneas tales como laboratorio e imágenes.

La Tabla 3 muestra factores de riesgo, etiología, clasificación Oxfordshire, puntaje de NIHSS y ASPECTS de ingreso de cada paciente.

$\mathrm{Al}$ ingreso dos pacientes (10,5\%) estaban en RANKIN 3, doce $(63,2 \%)$ en RANKIN 4 y cinco pacientes $(26,3 \%)$ en RANKIN 5.

$\mathrm{Al}$ momento del egreso, 15 pacientes $(78,9 \%)$ tenían un valor RANKIN menor o igual a 2 , dos RANKIN 3, uno RANKIN 4. Un paciente falleció al $6^{\circ}$ día posttrombolisis, evolucionando con infarto maligno de arteria cerebral media (Rankin 6). Hubo una hemorragia intracraneal $(5,2 \%)$ clínicamente catalogable como no significativa, y dos pacientes con gingivorragia menor.

A los 90 días de seguimiento, 12 pacientes (63\%) no presentaban discapacidad (Rankin 0-1); cinco pacientes estaban con discapacidad leve (Rankin 2) y uno en Rankin 3. En otras palabras, la evolución de los pacientes medida en escala de Rankin modificada muestra una favorable evolución entre el ingreso y los 90 días de evolución (Figura 3). La comparación de la condición de los pacientes entre el egreso y los 90 días muestra diferencias estadísticamente significativas $\mathrm{p}<0,001$ $\left(\chi^{2}=44,1 ; 12 \mathrm{gl}\right)$.

El análisis de la evolución del puntaje de NIHSS, también muestra diferencia estadísticamente significativa. Al ingreso, ningún paciente tenía puntaje NIHSS $0-5$, mientras que a las 24 horas, seis pacientes se encontraban en este rango, a los 30 días 14 y a los 90 días 17 (casi el 90\%) habían alcanzado dicho puntaje en la escala NIHSS.

Los datos muestran que la mejoría en la escala NIHSS entre los 30 días y los tres meses de seguimiento es estadísticamente significativa $\mathrm{p}<0,001$ $\left(\chi^{2}=18,2 \mathrm{gl}\right)$. La media del valor de NIHSS evoluciona desde el valor 16 al ingreso, 6 a las 24 
Trombolisis en infarto cerebral agudo - T. Figueroa-Reyes et al

Tabla 3. Descripción de factores de riesgo, etiología, clasificación Oxfordshire, puntajes de NIHSS y de ASPECTS

\begin{tabular}{|c|c|c|c|}
\hline & & $\mathbf{n}$ & $\%$ \\
\hline \multirow[t]{8}{*}{ Factores de riesgo } & HTA & 14 & 73,7 \\
\hline & Diabetes & 3 & 15,8 \\
\hline & Dislipidemia & 4 & 21,1 \\
\hline & Obesidad & 3 & 15,8 \\
\hline & Tabaquismo & 9 & 47,4 \\
\hline & Fibrilación auricular & 1 & 5,3 \\
\hline & Infarto cerebral previo (más de 1 año) & 1 & 5,3 \\
\hline & Cardiopatía coronaria & 4 & 21,1 \\
\hline \multirow[t]{5}{*}{ Etiología } & Cardioembólico & 5 & 26,3 \\
\hline & Aterotrombótico de grandes vasos & 9 & 47,4 \\
\hline & Aterotrombótico de pequeño vaso & 1 & 5,3 \\
\hline & Infarto de causa inhabitual & 1 & 5,3 \\
\hline & Infarto de origen no determinado & 3 & 15,7 \\
\hline \multirow[t]{5}{*}{ Clasificación Oxfordshire } & $\mathrm{PACl}$ & 8 & 42,1 \\
\hline & $\mathrm{POCl}$ & 4 & 21,1 \\
\hline & TACl & 5 & 26,3 \\
\hline & $\mathrm{LACl}$ & 1 & 5,3 \\
\hline & $\mathrm{TACl}$ y $\mathrm{POCl}$ & 1 & 5,3 \\
\hline \multirow[t]{12}{*}{ Valores de NIHHS al ingreso } & 11 & 4 & 21,1 \\
\hline & 12 & 1 & 5,3 \\
\hline & 14 & 3 & 15,8 \\
\hline & 15 & 1 & 5,3 \\
\hline & 16 & 2 & 10,5 \\
\hline & 17 & 1 & 5,3 \\
\hline & 18 & 1 & 5,3 \\
\hline & 19 & 1 & 5,3 \\
\hline & 20 & 2 & 10,5 \\
\hline & 23 & 1 & 5,3 \\
\hline & 24 & 1 & 5,3 \\
\hline & 33 & 1 & 5,3 \\
\hline \multirow[t]{3}{*}{ Puntaje ASPECTS al ingreso } & 8 & 6 & 31,6 \\
\hline & 9 & 10 & 52,6 \\
\hline & 10 & 3 & 15,8 \\
\hline
\end{tabular}




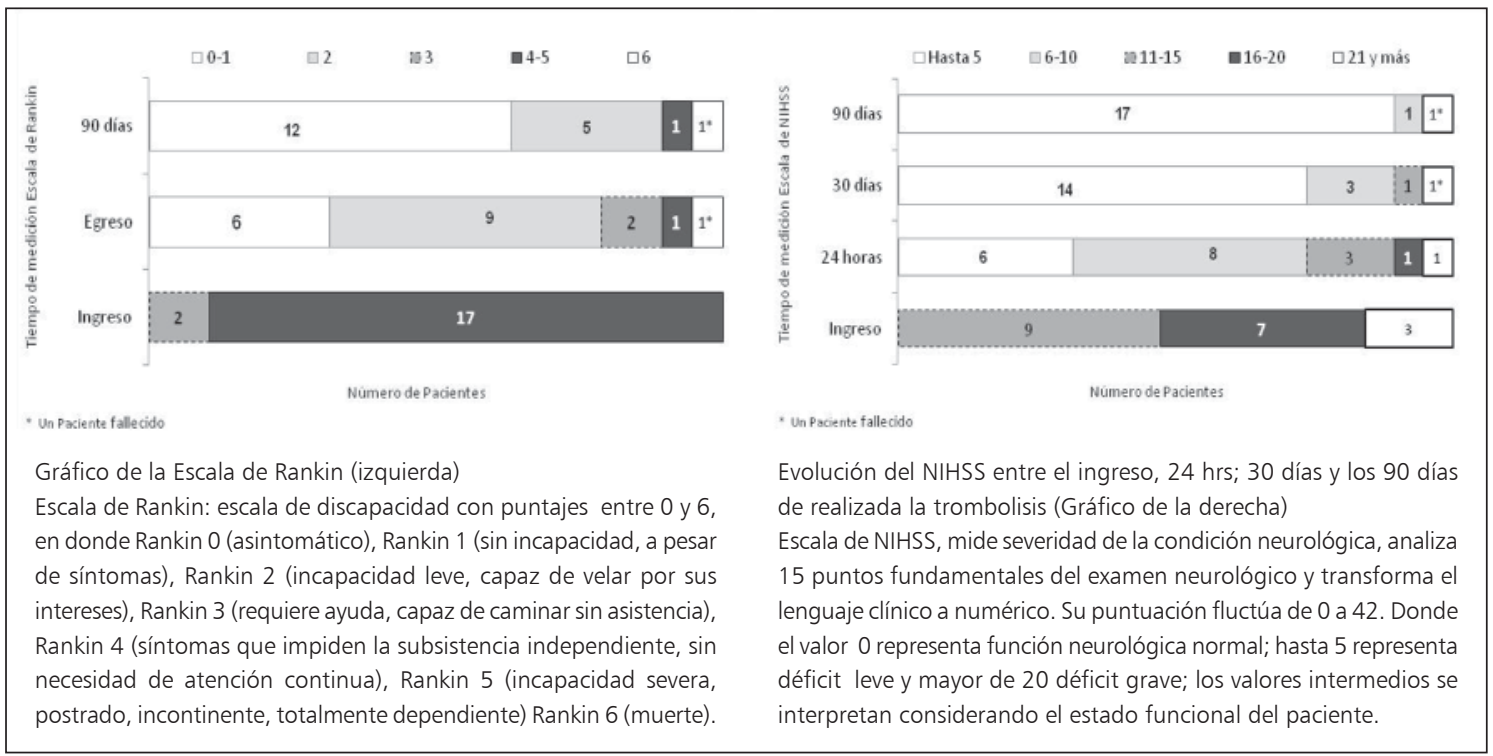

Figura 3. Evolución de Escala de Rankin Modificado y Escala de NIHSS.

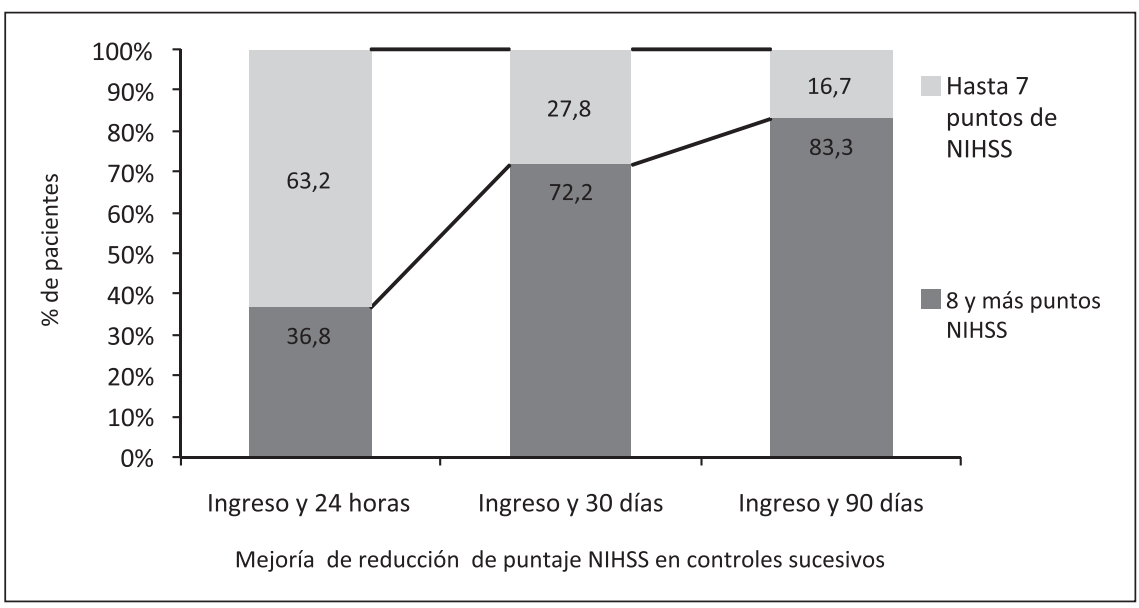

Figura 4. Reducción del puntaje de la Escala de NIHSS entre ingreso y los distintos controles. Mejoría medida en puntaje NIHSS en controles sucesivos. Dado que el puntaje NIHSS promedio de ingreso fue de 16 puntos, se destaca en este grafico que la mayoría de los pacientes $(83,3 \%)$ disminuyó 8 puntos o más después de la trombolisis. horas, 5 a los 30 días, hasta llegar a un valor de 3 al control de los tres meses.

Finalmente, se analizó la disminución del puntaje de NIHSS como variable dicotómica, hasta 7 puntos en la escala de NIHSS y 8 y más puntos, entre el ingreso y los distintos momentos; 24 horas; 30 y 90 días respectivamente.

En la Figura 4, se observa que, 12; 5 y 3 pacientes habían disminuido hasta 7 puntos de NIHSS, mientras que 7; 13 y 15 personas disminuyeron más de 8 puntos en las tres mediciones. El puntaje
NIHSS promedio al ingreso fue de 16; postrombolisis a los 90 días, la mayoría de los pacientes $(83,3 \%)$ evolucionaron hacia un puntaje NIHSS menor a 7, lo que se considera déficit neurológico leve ${ }^{25}$.

\section{Discusión}

La trombolisis con t-PA está probada y aceptada hasta las $4,5 \mathrm{~h}$ del inicio del evento isquémico ${ }^{8,9}$. 
Trombolisis en infarto cerebral agudo - T. Figueroa-Reyes et al

Si bien existe experiencia de trombolisis en Chile desde $1996^{23}$ y en otros países latinoamerica$\operatorname{nos}^{26-28}$, la mayoría de los centros que la realizan son clínicas del sector privado, en grandes zonas urbanas y hospitales universitarios. En Chile, la población usuaria del sector público no tenía hasta ahora acceso a esta terapia.

Los resultados presentados, son comparables a trabajos extranjeros y nacionales ${ }^{4-9,22}$, en términos de mortalidad, secuelas y puntajes del NIHSS entre otros. Estudios internacionales han demostrado una asociación entre la eficacia del tratamiento y el intervalo del tiempo transcurrido desde el inicio de los síntomas al inicio de trombolisis ${ }^{4-9,22}$, en nuestro trabajo la media de este intervalo fue 198 minutos, hubo una hemorragia intracraneal clínicamente no significativa. La mediana del NIHSS al ingreso fue de 16 puntos (promedio 16,53), la mortalidad 5,2\%. En ECASS III la media de inicio del procedimiento fue de 239 minutos, con $27 \%$ de hemorragias totales, $2,4 \%$ de las cuales fueron significativas, con una mediana de puntaje NIHSS de ingreso de 12 y la mortalidad fue de 7,7\%. Es necesario dejar en claro que nuestra muestra es pequeña.

Los aspectos mejorables para optimizar el intervalo de tiempo entre el inicio de síntomas y la trombolisis podría estar relacionado principalmente con aspectos tales como: 1) el paciente no consulta directamente a nuestro centro; 2) derivación desde otro centro asistencial hospitalario o de centro de atención primaria; 3 ) dificultad en el traslado; 4) demora en decisión de consultar; 5) tiempo de traslado desde SU a Neurología y 6) demora en certeza diagnóstica en dos casos de enclaustramiento.

Internacionalmente entre el 1 al $8 \%$ de los pacientes elegibles para trombolisis están siendo tratados ${ }^{24}$, según estimaciones del año 2000 para nuestro medio el 4\% de los pacientes llega en ventana $^{12}$, lo que no significa necesariamente que cumplan criterios aceptados para trombolisis. El número total de infartos egresados entre septiembre de 2009 y abril de 2010 fue de 390 pacientes. Nuestra serie de 19 pacientes trombolizados, en el mismo período correspondió al 4,9\%.

La fortaleza de estos resultados radica principalmente en que se muestra la aplicación óptima de un tratamiento de punta, en un hospital de red pública del sistema de salud chileno. Los principales obstáculos que habitualmente se esgrimen para su implementación son su costo y la carencia de infraestructura adecuada. Sin embargo, según lo observado en nuestra experiencia y para nuestra realidad, el factor central en la implementación de este procedimiento es la gestión orientada al recurso humano.

La implementación de la trombolisis significó mejorías en una serie de aspectos relacionados con estándares de calidad en nuestro quehacer, priorizando la atención del paciente con ECV agudo a su llegada al Servicio de Urgencia. Destaca la diligencia en el actuar de nuestros técnicos, profesionales y personal auxiliar para la pronta obtención de exámenes de laboratorio, TC cerebral y traslado de pacientes dentro del hospital. En este sentido la gestión debe enfocarse en la información, capacitación y motivación de todos los actores en este proceso.

Nuestros resultados indican que la trombolisis endovenosa es posible de realizar con éxito en la red pública, con cifras comparables a otras series. Aspiramos a su generalización en el contexto del sistema GES, de modo de acercar una oferta de calidad con equidad para toda la población del país.

Agradecimientos: A todo el equipo de enfermería de Servicio de Neurología y Urgencias, por su disciplina, dedicación y conocimiento, especialmente a: Viviana Herrera, por la confección de ficha, Claudia Figueroa, jefa de enfermería del Servicio, Víctor Manríquez, Cristian Castro, Sergio Díaz. A todos los técnicos paramédicos, por su lúcida capacidad de enfrentar nuevos desafíos: Sres. Marcos Orellana, Yasna Flores. Eduardo Erazo, Luis Varga, Ángela Oquendo, Miguel Baguerle, Odilia Manquepan, Carolina Villar, Margarita Andrade, Ivonne Romero, Roxana Astorga, Sonia Román, Ana Marín, Paola Briones. Nicole Muñoz, Maribel Kruger, al personal de laboratorio y scanner, al Dr. Luis Álvarez, por la adaptación de la escala de NIHSS, a nuestros becados por su exploración neurológica detallada y meticulosa. A nuestros neurólogos residentes, por su excelencia en el diagnóstico clínico y su compromiso por hacer neurología de primer nivel: Dres. Andrés Aragón, Rodrigo Aracena, Braulio Troncoso. A la Dirección de nuestro establecimiento que creyó en el proyecto y en la posibilidad de lograrlo, al Sr. Vicente Zúñiga por su contribución a la idea general del artículo y a todos nuestros pacientes. 


\section{Referencias}

1. Lavados P, Sacks C, Prina L, Escobar A, Tossi C, Araya $\mathrm{F}$, et al. Incidence, case fatality rate, and prognosis of ischaemic stroke subtypes in a predominantly HispanicMestizo population in Iquique, Chile (PISCIS project): a community based incidence study .The Lancet Neurology 2007; 6: 140-8.

2. Ministerio de Salud de Chile. Base de datos de egresos hospitalarios, departamento de estadística. Consultado el 5 de junio 2010, disponible en URL: http://deis.minsal.cl

3. Ministerio de Salud Informe final estudio de Carga de Enfermedad y Carga Atribuible, Documento Minsal, Chile 2007.

4. The National Institute of Neurological Disorders and Stroke rt-PA Stroke Study Group. Tissue plasminogen activator for acute ischemic stroke. N Engl J Med 1995; 333: 1581-7.

5. Hacke W, Kaste M, Fieschi C, Toni D, Lesaffre E, von Kummer R, et al. Intravenous thrombolysis with recombinant tissue plasminogen activator for acute hemispheric stroke: the European Cooperative Acute Stroke Study (ECASS). JAMA 1995; 274: 1017-25.

6. Hacke W, Kaste M, Bogousslavsky J, Brainin M, Chamorro A, European Stroke Initiative Executive Committee and the EUSI Writing Committee. European Stroke Initiative Recommendations for Stroke Managementupdate 2003. Cerebrovasc Dis 2003; 16: 311-37.

7. Marler JR, Tilley BC, Lu M, Brott TG, Lyden PC, Grotta JC, et al. Early stroke treatment associated with better outcome: the NINDS rt-PA Stroke Study. Neurology 2000; 55: 1649-55.

8. Bluhmki E, Chamorro A, Dávalos A, Machnig TH, Sauce C, Wahlgren N, et al. Stroke treatment with Alteplase given 3.0-4.5 h after onset of acute ischaemic stroke (ECASS III): additional outcomes and subgroup analysis of a randomized controlled trial. Lancet Neurol 2009; 8: 1095-102.

9. Hacke W, Kaste M, Bluhmki E, Brozman M, Dávalos A, Guidetti D, et al, for the ECASS Investigators Thrombolysis with Alteplase 3 to 4.5 hours after acute ischemic stroke. N Engl J Med 2008; 359: 1317-29.

10. Guía para el manejo del infarto cerebral agudo. Consultado el 15 de mayo 2010. Disponible en URL: www. minsal.cl/guías.

11. Nogales-Gaete J. Propuesta para la atención de pacientes adultos, con Enfermedad Cerebro Vascular en etapa aguda, en el Hospital Barros Luco Trudeau, Servicio de Salud Metropolitano Sur. Autoedición, Santiago, Chile, 1998.
12. Nogales-Gaete J, Núñez L, Arriagada C, Sáez D, Figueroa T, Fernández R, et al. Caracterización clínica de 450 pacientes con enfermedad cerebrovascular ingresados a un hospital público durante 1997. Rev Med Chile 2000; 128: 1227-36.

13. Kothari RU, Pancioli A, Liu T, Brott T, Broderick J. Cincinnati Prehospital Stroke Scale: reproducibility and validity. Ann Emerg Med 1999; 33 (4): 373-8.

14. Montaner J, Álvarez-Sabin J. La escala de Ictus del National Institute of Health (NIHSS) y su adaptación al español. Neurología 2006; 21(4): 192-202.

15. Brott T, Adams HP Jr, Olinger CP, Marler JR, Barsan WG, Biller J, et al. Measurements of acute cerebral infarction: a clinical examination scale. Stroke 1989; 20: 864-70.

16. Lin K, Rapalino O, Law M, Babb JS, Siller KA, Pramanik BK. Accuracy of the Alberta Stroke Program Early CT Score during the First 3 Hours of Middle Cerebral Artery Stroke: Comparison of Noncontrast CT, CT Angiography Source Images, and CT Perfusion. AJNR Am J Neuroradiol 2008; 29: 931-6.

17. Weir NU, Pexman JH, Hill MD, Buchan AM; CASES investigators. How well does ASPECTS predict the outcome of acute stroke treated with IV tPA? Neurology 2006; 67: 516-8.

18. Pexman JHW, Barber PA, Hill M, Sevick RJ, Demchuk AM, Hudon ME, et al. Use of the Alberta Stroke Program Early CT Score (ASPECTS) for Assessing CT Scans in Patients with Acute Stroke. AJNR Am J Neuroradiol 2001; 22: 1534-42.

19. Van Swieten JC, Koudstaal PJ, Visser MC, Schouten HJ, van Gijn J. Interobserver agreement for the assessment of handicap in stroke patients. Stroke 1988; 19 (5): 6047.

20. Adams HP, Bendixen BH, Kappelle LJ, Biller J, Love BB, Gordon DL, et al. Classification of subtype of acute ischemic stroke: definitions for use in a multicenter clinical trial. Stroke 1993; 24: 35-41.

21. Bamford J, Sandercock PA, Dennis MS, Burn J, Warlow CP. Classification and natural history of clinically identifiable subtypes of brain infarction. Lancet 1991; 337: 1521-6.

22. The European Stroke Organisation (ESO) Executive Committee. Guidelines for management of ischaemic stroke and transient ischaemic attack 2008. Cerebrovasc Dis 2008; 25: 457-507.

23. Feuerhake W, Chamorro H, Araya F. Activador del plasminógeno tisular intravenoso en el tratamiento del infarto cerebral agudo: Factibilidad, seguridad y eficacia en los primeros dos años de práctica clínica. Rev Med Chile 1999; 127: 814-9. 
24. Reeves MJ, Arora S, Broderick JP, Frankel M, Heinrich JP, Hickenbottom S, et al; Paul Coverdell Prototype Registries Writing Group. Acute stroke care in the US: results from the 4 pilot prototypes of the Paul Coverdell National Acute Stroke Registry. Stroke 2005; 36: 1232-40.

25. Ji-Sun Kim MD, Kyung-Bok Lee MD, Hakjae Roh MD, Moo-Young Ahn MD, Hye-Won Hwang. Gender Differences in the Functional Recovery after Acute Stroke RN. J Clin Neurol 2010; 6: 183-8.

26. Durai Pandian J, Padma V, Vijaya P, Sylaja PN, Murthy
JM. Stroke and thrombolysis in developing countries. Int J Stroke 2007; 2: 17-26.

27. Sposato LA, Esnaola MM, Zamora R, Zurrú MC, Fustinoni O, Saposnik G, et al. Quality of Ischemic Stroke Care in Emerging Countries The Argentinian National Stroke Registry (ReNACer). Stroke 2008; 39: 3036-41.

28. Conforto AB, Paulo RB, Patroclo CB, Pereira SL, Miyahara Hde S, Fonseca CB, et al. Stroke management in a university hospital in the largest South American city. Arq Neuropsiquiatr 2008; 66: 308-11. 